\title{
Science on WeChat
}

\author{
We distributed an informal questionnaire to learn from scientists about their professional use of WeChat. We share \\ some of their answers and discuss the multipurpose platform offered by WeChat.
}

$\mathrm{S}$ ocial media has becomes an inevitable part of daily life. In our June editorial we conducted an informal survey about 'science Twitter' and discussed how actively tweeting can be beneficial for scientists. The number of daily active Twitter users, estimated to be around 145 million, is dwarfed by the estimated 1 billion daily active users of WeChat, the most widely used social media platform in China. WeChat, like Twitter, is a valuable tool for scientists to network, catch up on news, and cultivate collaborations.

WeChat, or Weixin, was initially developed as an instant messaging app launched in 2011 and later evolved into a multipurpose app through its integration with a social media platform, mobile payment, e-commerce shopping and mini program for local services. The messaging function makes WeChat a ubiquitous daily use app.

The ubiquity of WeChat is also mirrored in its use by Chinese scientists working both inside and outside China. In a 2019 Springer Nature survey, $94 \%$ of 528 respondents in China indicated that they had used WeChat in a professional context. The popularity of WeChat is rooted in its messaging function, which allows text and photo messages, as well as voice and video calls with individuals or a group of people.

Besides messaging, scientists on WeChat can also discover new content via subscription feed or 'Moments' sharing, which are channeled by two types of WeChat accounts: public accounts and personal accounts. Public or official accounts are often registered by an organization or enterprise and allow them to push feeds to subscribers. For example, Nature Research has its own official account. Some popular public accounts have gained over 1 million subscribers and are considered key opinion leaders in a specific field. Given the large number of subscribers, the distribution channel offered by official accounts can help researchers to promote their profiles and research activities to a broader audience.

Official accounts of scientific relevance often post original articles or research paper recommendations. The articles can range from science-related news and commentary to digest of published research papers to job listings and policy updates. In contrast, personal accounts, registered by an ID-verified phone number, can share
Moments with their contact network. A Moment can be text, images, video, podcast or article. In most cases, the shared articles are published by an official account. Users can elect to share Moments in a private or public manner, allowing the separation of professional activities from personal ones.

As we witnessed the expansion of professional use of WeChat in academia in China, we became interested in hearing thoughts from researchers about the benefits and concerns of using WeChat as a scientific social platform. We distributed a questionnaire to Nature Methods readers via e-mail, Twitter and the Nature Research WeChat account. We received 30 responses, from which we are not drawing quantitative conclusions about science on WeChat but rather offering a point of view about how our respondents perceive WeChat as a scientific social media platform.

All respondents are from academia and the majority are Chinese researchers who work in the biological sciences in China or the United States. More than half have been using WeChat for scientific activities for more than two years. The majority of respondents spend less than one hour a day on WeChat and do not consider WeChat a time sink, though one respondent noted that inessential chatting can be a waste of time.

Since WeChat is heavily integrated with daily tasks such as mobile payment, most scientists who responded use one WeChat account for both general and professional use. A small portion of our respondents indicated that they exclusively separate their personal and professional activities, but none of the respondents had strong concerns about blending personal and professional activities.

The respondents subscribe to a varying number of public accounts, ranging from a few to more than 100 , and most of our respondents follow more than 10 science-relevant public accounts. One researcher commented that official accounts are a good way to highlight new results and to stimulate further discussion in the community.

We also learned that although the public accounts can provide rapid scientific news updates, fewer than $20 \%$ of respondents use WeChat as their main news source. Most respondents browse journal websites and subscribe to email alerts when a topic is highly relevant. Our respondents think the articles posted in WeChat are generally reliable, yet they also indicate that they like to make their own judgments and often read the original research papers when they find a paper of interest.

We noted that group chat is also popular in Chinese academia as it offers an opportunity to group researchers and circulate ideas and questions to researchers working in the same field. Group chat enables crowdsourcing answers to technical questions, as well as connecting to new people within the group.

Though WeChat offers convenient communication, several researchers note that collaborations are not necessarily initiated under the WeChat umbrella, but are further developed via WeChat group chat and Moments sharing. Several respondents mention that WeChat has helped to speed up and nurture collaborations, particularly for Chinese-speaking scientists.

Since two members of our editorial team are based in China, we also asked our respondents whether they would like to communicate with editors on WeChat. About one-third respondents prefer to conduct formal communication via e-mail while the rest consider WeChat to be a more efficient communication tool. Our two China-based editors also find WeChat provides a convenient means for informal chats with authors and offers an alternative news outlet, particularly regarding general research trends, conference information and policy updates in China. However, we do not encourage authors to submit manuscripts via WeChat messaging; we instead ask to be contacted via e-mail or via the journal submission system.

The popularity of WeChat is apparent in the Chinese scientific community. Yet we recognize that this popularity may not extend worldwide, which may be partly because most WeChat content is written in Chinese, as well as because WeChat use is restricted in some countries. During the writing of this editorial, the Trump administration in the United States issued two orders banning transactions with WeChat and TikTok. Although the scope of the ban remains blurry, we hope that science communication and collaboration built on the WeChat platform across the world will not be disrupted and will remain unsullied by government influence.

Published online: 1 September 2020 https://doi.org/10.1038/s41592-020-0954-1 